\title{
Distribución Espacial y Actores Privilegiados en la Ley de Riego: Análisis Comunal de la Región de O’Higgins
}

\author{
Spatial Distribution and Privileged Actors in the Irrigation Law: \\ Community Analysis of the O'Higgins Region
}

\begin{abstract}
Marco Pavez*
Resumen: El presente artículo analiza el gasto público en el territorio asociado a la ley $\mathrm{N}^{\circ}$ 18.450 , de fomento a la inversión privada en obras de riego y drenaje, develando la formación de polos de asignación heterogénea en las comunas de la región de O’Higgins. Se destaca el impulso a la producción de medianos y grandes productores insertos en los mercados domésticos e internacionales, en contraste al insuficiente aporte a la agricultura de menor escala, en donde se concentra históricamente la pobreza de la zona centro sur agrícola y forestal. En particular, la apuesta nacional de las últimas décadas por posicionarse como una potencia agroexportadora se ha traducido en el aumento tanto de la concentración de grandes empresas frutícolas, forestales y vinícolas como también de conflictos territoriales, entre los que se encuentran la seguridad hídrica para el consumo humano y el uso de cerros para la expansión de monocultivos de paltas, viñas, cerezos y olivo. El artículo logra caracterizar la tipología de actores beneficiados, zonificando la envergadura de las asignaciones en las comunas de la región de O'Higgins. Al revisar los montos, se observa el privilegio en la asignación de recursos y la ausencia de una política mayor que articule el apoyo a los pequeños productores, contradiciendo los lemas de crecimiento con equidad del Ministerio de Agricultura.
\end{abstract}

Palabras clave: Distribución espacial, ley $\mathrm{N}^{\circ}$ 18.450, concentración de asignaciones.

\begin{abstract}
The article analizes the local public expenditure of the law $\mathrm{N}^{\circ} 18.450$, to promote private investment in irrigation and drainage constructions, unveiling the formation of heterogeneous allocation poles in the communes of the O'Higgins region. The boost to the production of medium and large producers inserted in domestic and international markets stands out, in contrast to the insufficient contribution to small-scale agriculture, where poverty is historically concentrated. In particular, the national commitment in recent decades to position itself as an agro-export country has resulted in an increase in both the concentration of large fruit, forestry and wine companies as well as territorial conflicts, among which are water security for the human consumption and the use of hills for the expansion of monocultures of avocados, vineyards, cherry trees and olive trees. The research manages to characterize the typology of benefited actors, zoning the size of the assignments in the communes of the O'Higgins region. When reviewing the amounts, it is observed the privilege in the allocation of resources and the absence of a greater policy that articulates the support to small producers, contradicting the slogans of growth with equity of the Ministerio de Agricultura.
\end{abstract}

Keywords: Spatial distribution, law $\mathrm{N}^{\circ}$. 18.450, allocation concentration.

Recibido: 13 enero 2021 Aceptado: 22 abril 2021

\footnotetext{
* Licenciado en Administración Pública y Ciencia Política en la Universidad de Concepción. Investigador asociado de Crea-Sur UdeC. E-mail de contacto: marcopz03@outlook.com.
} 


\section{Introducción}

Mientras la instauración del neoliberalismo en Chile ha logrado un tremendo crecimiento económico en las tres últimas décadas, la desigualdad entre los territorios del país se ha profundizado. Esta disonancia se ha traducido en una demanda histórica de los movimientos regionalistas o federalistas del país, junto con ser un elemento central tanto en la promulgación de políticas asociadas ${ }^{1}$ como en la escrituración de una nueva Constitución.

Una parte de la geografía económica dilucida la desigualdad territorial en torno a la concentración de las actividades económicas. La teoría de los polos de crecimiento (Boudeville, 1967; Hirschman \& Sirkin, 1958; Lasuen, 1969; Perroux, 1950, 1955) estudia los vínculos de colaboración y mutuo beneficio entre actores de un rubro junto a la vinculación de sus resultados con el territorio. En la actualidad, el enfoque de clústeres (Porter, 1990, 1995, 1996, 2000) ha desplazado los estudios en torno a los polos de crecimiento, por lo que se vuelve necesaria su consideración en el presente artículo.

En específico, la apuesta nacional de las últimas décadas por posicionarse como una potencia agroexportadora ha tenido efectos disonantes. Por un lado, las políticas de fomento y clusterización ${ }^{2}$, como también el aumento del presupuesto afín, se han relacionado con el aumento del PIB del rubro (Oficina de Estudios y Políticas Agrarias [ODEPA], 2019) y en una mejora en el salario de los trabajadores (Comisión Económica para América Latina y el Caribe [CEPAL], 2010). Por otro, ha causado la concentración de empresas frutícolas, forestales y vinícolas en desmedro de la agricultura familiar y pequeña agricultura junto con el aumento de conflictos territoriales ${ }^{3}$. Asimismo, según la encuesta CASEN del año 2017, la incidencia de la pobreza, extrema pobreza y pobreza multidimensional en la población rural fue del 16,5\%,4,4\%, y 37,4\% respectivamente, evidenciando una parte de las brechas territoriales persistentes.

Ya que la literatura se especializa en el estudio de ventajas, beneficios y modelos de perfectibilidad de los polos, son menores aquellas investigaciones relacionadas con los efectos negativos del mismo. En este sentido, destacan la investigación de Chapman (2005), quien analiza la evolución, las dinámicas de aglomeración y las políticas influyentes en el clúster de la industria química en Teesside, al noreste de Inglaterra, evidenciando su incidencia en el bloqueo territorial provocado por la limitada permeabilidad del crecimiento sobre el resto empresas y el territorio. Por su parte, Smékalová et al. (2014) analizan la política eslovaca de polos de crecimiento, a la vez que los clasifican en tipos. Concluyen que se les asigna mayor cantidad de recursos a aquellos polos ubicados en los municipios que concentran las actividades económicas del país. Wiesel et al. (2018) estudian la desigualdad territorial resultante de la distribución espacial del gasto público en infraestructura urbana y en servicios en la zona metropolitana de Sidney, reconociendo los territorios que resultan beneficiados y en desventaja.

\footnotetext{
${ }^{1}$ En cuanto a la descentralización política, destaca la reforma constitucional producto de la ley $\mathrm{N}^{\circ} 20.990$ en el año 2017, que dispone la elección popular directa de los gobernadores regionales, y la ley $\mathrm{N}^{\circ} 21.073$, del 2018, que regula dicho proceso. Respecto a la descentralización administrativa, la ley $\mathrm{N}^{\circ} 21.074$, del mismo año, busca el fortalecimiento regional mediante el traspaso de competencias asociadas al ordenamiento territorial, el fomento productivo y el desarrollo social y cultural. Asimismo, destaca la promulgación de la Política Nacional de Desarrollo Rural (PNDR) a través del Decreto $\mathrm{N}^{\circ} 19$, en el año 2020 , la cual busca articular los componentes territoriales de las zonas rurales y avanzar en un nuevo paradigma de "lo rural". ${ }^{2}$ Véase el clúster de la palta (Figueroa-Sterquel et al., 2016) o, fuera de la agricultura, la promoción de la industria forestal y silvícola. Incluso podría considerarse el caso de la Ciudad Empresarial en el sector nororiente de Santiago. Como se indicará a lo largo del documento, si bien se cumple la disposición relacionada con la aglomeración espacial, se postula que no existe la articulación ni coordinación necesaria para mejorar las propias condiciones.

${ }_{3}^{3}$ Destacan los casos de falta de seguridad hídrica para el consumo humano en algunas comunas del país o la expansión indiscriminada de monocultivos de paltas, viñas, cerezos y olivos hacia cerros que cuentan con vegetación esclerófila y xerófila endémica de protección, entre otros.
} 
En base a los anteriores párrafos, el presente artículo tiene por objetivo analizar los vínculos generados a raíz de las asignaciones por concepto de la ley $\mathrm{N}^{\circ} 18.450$, o ley de riego. Se delimita la investigación en torno a las comunas de la región de O’Higgins y a las transferencias ocurridas entre los años 1990 y 2019. En específico, se estudia la formación de patrones y dinámicas en las transferencias monetarias, distinguiendo entre sus destinos geográficos, la envergadura de los montos, la tipología de los productores beneficiarios y la plausible desigualdad generada a raíz de ellas. Mediante el análisis de las transferencias y su visualización en mapas coropléticos ${ }^{4}$, se tiene que la distribución del presupuesto de la ley $\mathrm{N}^{\circ} 18.450$ se encuentra altamente concentrada en 2 de las 33 comunas de la región, evidenciando la formación de polos de alta y baja asignación presupuestaria junto con estudiar las implicancias de ello.

En cuanto a la estructura del artículo, en su primera sección se revisa someramente la literatura asociada a los polos de crecimiento, avanzando hacia el enfoque basado en clústeres. La segunda sección contiene una reseña de la ley de riego, indicando la estrategia subyacente a su diseño. Además, se delimita el objeto de estudio a la clasificación de productores establecida en la Resolución Exenta $\mathrm{N}^{\circ}$ 4.954 de la CNR, la cual servirá para reconocer los receptores del subsidio. La tercera sección detalla la metodología a seguir en el desarrollo de la investigación. La cuarta sección presenta el contraste y la discusión entre los resultados obtenidos y la teoría presentada, mientras que, por último, la quinta sección sintetiza los aspectos más relevantes del artículo, a modo de conclusión.

\section{Revisión de la literatura}

La teoría de los polos de crecimiento surge en la década de 1950 a raíz del trabajo de François Perroux, quien, desde la geografía económica, buscó explicar parte de las dinámicas del desarrollo territorial. Concretamente, el concepto refiere al vínculo formado entre empresas e industrias ${ }^{5}$, bajo las cuales subyace la premisa de dominación de unas sobre otras (Perroux, 1955). Las características de sus relaciones se basan en la asimetría e irreversibilidad, en la medida que la empresa dominante es aquella que dinamiza el crecimiento de la industria, imponiendo condiciones para la apropiación de excedentes, los que refuerzan su dominio y, según el planteamiento de Myrdal (1957), permean, en menor medida, al resto de actores y territorio, fenómeno conocido como spread-backwash effect.

En particular, la relación entre las industrias y el Estado representa un actuar simultáneo para el establecimiento de unidades capaces de favorecer los intereses de ambos actores. Este crecimiento no es un proceso homogéneo en cuanto a expresión espacial, temporal o incluso de intensidad, más bien suele ser concentrado en el territorio (Boudeville, 1967), intermitente en el tiempo y desigual entre los actores $^{6}$ (Hirschman \& Sirkin, 1958).

Con todo, es de esperar que la concentración de estas actividades tenga resultados favorables en cuanto a la calidad de los bienes o servicios, el precio de los insumos necesarios para su producción o en la innovación de los procesos productivos asociados. Sin embargo, si bien las políticas orientadas a la

\footnotetext{
${ }^{4}$ Como indica la Escuela Técnica Superior de Ingenieros en Topografía, Geodesia y Cartografía de la Universidad Politécnica de Madrid, los mapas coropléticos son "una forma de cartografiado cuantitativo utilizada para la representación de fenómenos discretos asociados a unidades de enumeración (provincias, países) a las que se aplican símbolos superficiales de acuerdo con su valor", a lo que agrega que "normalmente se realizan para obtener una idea de la distribución general de la variable cartografiada, y su función más importante suele ser la de comparar unos mapas con otros" (UPM, s. f.).

${ }^{5}$ Comprendiendo a la empresa como una entidad cuya actividad se basa en la producción de bienes o la prestación de servicios y a la industria como el conjunto de operaciones realizadas por empresas del mismo rubro. Por ello, la empresa se asocia frecuentemente a una personalidad jurídica o natural, mientras que la industria se asocia a un rubro o actividad. La industria agrícola, láctea o forestal son algunos ejemplos.

${ }^{6}$ Los párrafos anteriores dilucidan el contenido del término polo de crecimiento. Sin embargo, en la teoría no se encuentra del todo claro, ya que el mismo Perroux suele contradecir el significado de sus planteamientos. Como acredita Coraggio (1972), esta es una de las críticas esenciales recibidas por esta teoría, lo que causó el cese de sus estudios en los años 60-70.
} 
construcción de dichos polos incidieron en la diversificación de los productos al trabajar la cadena de suministros y profundizaron las relaciones basadas en la información y el conocimiento, promovían, en la práctica, la especialización productiva en el territorio (Chapman, 2005). Lo anterior por cuanto la empresa dominante pudiese retener el crecimiento de la industria, dificultando la permeabilidad de los beneficios sobre el resto de empresas y del territorio, fenómeno llamado locked-in.

En la actualidad, el enfoque de clústeres es el equivalente a los polos de crecimiento (Martin \& Sunley, 2003). Dicho enfoque es atribuido a Michael Porter (1990), quien considera que las ventajas comparativas de los países, junto con ser condiciones naturales y propias al territorio, derivan de una mejoría en la productividad ganada a través del continuo desarrollo de la innovación y calidad de los procesos productivos. A diferencia de Perroux, Porter si entrega una definición acerca de lo que concibe como un clúster, al declarar que estos:

Son concentraciones geográficas de empresas e instituciones interconectadas en un campo o área en particular. Abarcan una variedad de industrias vinculadas y otras entidades importantes para la competencia. Incluyen, por ejemplo, proveedores de insumos necesarios para el funcionamiento del clúster, como componentes, maquinaria, servicios o proveedores de infraestructura especializada. Los clústeres también se extienden, a menudo, hacia pequeños negocios y clientes, como también a los fabricantes de productos complementarios y a las empresas relacionadas, ello en cuanto a habilidades, tecnologías o insumos comunes.

Por último, muchos de ellos incluyen instituciones gubernamentales o de otro tipo, como universidades, agencias de establecimiento de estándares, think tanks, proveedores de formación profesional o asociaciones comerciales, las que proporcionan formación especializada, educación, información, investigación y apoyo técnico. (Traducción propia desde Porter, 1998, p. 78)

En este sentido, mientras Perroux ponía su atención en las lógicas de dominación entre empresas, Porter lo hace sobre las lógicas de cooperación originadas no sólo por la aglomeración de empresas en una zona geográfica, sino que también fuera de los límites territoriales de la industria, asumiendo la integración internacional de las economías domésticas, como también la vinculación con instituciones relacionadas al sector cuaternario de la economía, esto es, a entidades avocadas a la generación de conocimiento e información. Asimismo, el enfoque de los clústeres tiende a priorizar la influencia de las pequeñas y medianas empresas en los procesos de innovación y crecimiento económico a escala territorial, sin descartar la construcción de clústeres en torno a grandes empresas. En este sentido, la definición indica que existe una vinculación vertical entre la cadena industrial y los clientes y una vinculación horizontal entre la industria, sus relacionadas y aquellas que, no siendo afines, le permiten potenciar su crecimiento.

\section{Políticas agrarias: ley de riego en la región de O’Higgins}

Para lograr posicionar a nuestro país como una potencia agroexportadora, el rubro se ha visto beneficiado por diversas políticas de incentivo, promoción e inversión. En esta línea, estudios de la ODEPA (2019) y, en el caso de la región latinoamericana, los estudios de Anríquez et al. (2016) y del Banco Mundial (2005), evidenciaron que gran parte del gasto público agrícola se destinaba, mayoritariamente, a bienes y transferencias privadas, lo que no generaba retornos sociales, laborales, tecnológicos o pecuniarios.

Una de dichas políticas ha sido la ley $\mathrm{N}^{\circ} 18.450$, de fomento a la inversión privada en obras de riego y drenaje, cuya gestión y ejecución se encuentra a cargo de la CNR. Sus principales objetivos refieren incrementar la superficie regada del país, mejorar el abastecimiento de agua e incentivar un uso 
más eficiente del recurso. Modificaciones recientes de la norma -mediante la ley $\mathrm{N}^{\circ} 20.705$ y el Decreto $\mathrm{N}^{\circ} 95$ del MINAGRI- ampliaron el tramo que el costo de los proyectos a postular pueden tener llegando a 50.000 UF en el caso de cualquier persona natural o jurídica y a $250.000 \mathrm{UF}$ en el caso de las organizaciones- y promovió la adjudicación de obras integrales y de uso múltiple (Biblioteca del Congreso Nacional [BCN], 2018). Algunas de las iniciativas que sustentaban dicha modificación refieren a la dinamización de las asociaciones de productores, la recuperación de la calidad de aguas contaminadas y el fomento al uso y generación de energías renovables no convencionales. En definitiva, su diseño se orienta a suplir la escasa infraestructura en el agro, siguiendo una estrategia de intensificación en los factores productivos 7 .

Es de interés estudiar dicha política bajo los supuestos de este artículo ya que, en primer lugar, implica una transferencia monetaria directa como tal, esto es, cuenta, en primer lugar, con un receptor concreto -una persona natural o jurídica cuyo destino geográfico es expresable en un mapa- y con un monto de transferencia establecido, el cual es medible y cuantificable. En segundo lugar, debido a que su presupuesto representa el 83,04\% del total del financiamiento de la CNR, implicando el grueso de las funciones del servicio. La tabla 1 reúne los estratos de los productores utilizados por la ley $\mathrm{N}^{\circ} 18.450$, cuya definición y especificaciones se encuentran en el Manual de Procedimiento legal-administrativo de la CNR, establecido por la Resolución Exenta Nº 4.954 del 2019.

Tabla 1 Estrato de productores establecidos en la ley $\mathrm{N}^{\circ} 18.450$

\begin{tabular}{|c|c|}
\hline Clasificación de productores & Especificaciones de la persona natural o jurídica \\
\hline Productor INDAP & $\begin{array}{l}\text { - Explota una superficie no mayor a las } 12 \text { hectáreas de riego } \\
\text { básicoa } \\
\text { - Activos no superen el equivalente a } 3.500 \mathrm{UF} \\
\text { - Ingresos provenientes principalmente de la explotación agrícola } \\
\text { - Debe trabajar directamente la tierra, cualquiera sea su régimen de } \\
\text { tenencia } \\
\text { - Podrán optar a una bonificación máxima del } 90 \% \text {. }\end{array}$ \\
\hline Pequeño empresario & $\begin{array}{l}\text { - Poseen predios cuyas superficies físicas de riego convertidas a } \\
\text { hectáreas de riego ponderadas son de hasta } 40 \\
\text { - Podrán optar a una bonificación máxima del } 80 \%\end{array}$ \\
\hline Empresario mediano & $\begin{array}{l}\text { - Poseen predios cuyas superficies físicas de riego convertidas a } \\
\text { hectáreas de riego ponderadas son mayores a } 40 \text { y hasta de } 200 \\
\text { - Podrán optar a una bonificación máxima del } 70 \% \text {. }\end{array}$ \\
\hline Gran Empresario & $\begin{array}{l}\text { - Poseen predios cuyas superficies físicas de riego convertidas a } \\
\text { hectáreas de riego ponderadas son mayores a } 200 \\
\text { - Podrán optar a una bonificación máxima del } 70 \% \text {. }\end{array}$ \\
\hline $\begin{array}{l}\text { Organizaciones de Pequeños } \\
\text { Usuarios del Agua (OPUA) }\end{array}$ & $\begin{array}{l}\text { - Aquellas organizaciones en que al menos el } 70 \% \text { de sus } \\
\text { integrantes son Productores INDAP y/o Pequeños Empresarios } \\
\text { - Podrán optar a una bonificación máxima del } 90 \% \text {. }\end{array}$ \\
\hline $\begin{array}{l}\text { Organizaciones de Usuarios del } \\
\text { Agua (OUA) }\end{array}$ & $\begin{array}{l}\text { - Aquellas organizaciones en que menos del } 70 \% \text { de sus } \\
\text { integrantes son pequeños productores agrícolas INDAP o } \\
\text { pequeños empresarios } \\
\text { - Podrán optar a una bonificación máxima del } 80 \%\end{array}$ \\
\hline
\end{tabular}

${ }^{7}$ En economía, los factores de producción corresponden la tierra, el trabajo, el capital y la tecnología. 
a Refiere a una unidad de superficie distinta a las hectáreas. Para su cálculo se utiliza la tabla de equivalencia de hectáreas físicas a hectáreas de riego básico establecida en el artículo 13 de la ley $\mathrm{N}^{\circ}$ 18.910, que sustituye la ley orgánica del Instituto de Desarrollo Agropecuario.

Respecto a la región de O’Higgins, su localización se encuentra en la macrozona central del país, limitando al norte con la región Metropolitana, al sur con la región del Maule, al oeste con el Océano Pacífico y al este con la Cordillera de Los Andes. Los factores climatológicos implican que posea un clima templado de tipo mediterráneo, el cual, junto con su sistema hidrográfico, otorgan un ambiente ideal para el ejercicio de la agricultura, principal actividad económica de la zona. Asimismo, se caracteriza por albergar industrias alimentarias y mineras. En este sentido, la región destaca por ser uno de los pilares en la estrategia por imponer a nuestro país como una potencia agroalimentaria a nivel internacional, junto con albergar a gran parte de agricultores insertos tanto en el mercado nacional como internacional, destacando en torno a la producción frutícola y de vides.

\section{Metodología}

La presente investigación es de tipo descriptiva y su enfoque es cuantitativo. Lo anterior por cuanto comprende y busca caracterizar la realidad delimitada a través de datos numéricos, postulando leyes universales bajo una lógica inductiva, ello con el fin de evidenciar la distribución territorial del presupuesto de la ley $\mathrm{N}^{\circ} 18.450$ junto con sus implicancias. Se hará uso tanto de fuentes primarias y secundarias, cuyo sustento corresponde a documentos elaborados tanto por instituciones estatales nacionales como por organismos internacionales y a artículos de distintas revistas científicas de renombre.

La recolección de los datos a utilizar se realizó a través del Portal de Transparencia, en el marco de la ley $\mathrm{N}^{\circ} 20.285$, sobre acceso a la información pública. La solicitud de transparencia dirigida a la CNR, con folio $\mathrm{N}^{\circ}$ AR002T0001380, resultó en una base de datos que alberga 24.264 observaciones a nivel nacional, y refiere a las transferencias destinadas a los productores agrícolas que se adjudicaron una obra contemplada por la ley $\mathrm{N}^{\circ} 18.450$ entre los años 1990 al 2019. Se detalla cada obra respecto a: su nombre, código y fecha de ingreso; el nombre, región, provincia y comuna del beneficiario; el consultor a cargo de la obra; el estrato o clasificación del productor beneficiario; el tipo de obra ${ }^{8}$; la superficie del predio del beneficiario y la superficie beneficiada por el proyecto; la fecha del pago, el monto del subsidio estatal en Unidad de Fomento (UF) y el costo total del proyecto en UF.

Su tratamiento se realizó mediante los softwares libres R y RStudio. El procedimiento se basó en la utilización de los paquetes estadísticos tidyverse, sf, geofacet y ggpubr, no restringiendo el uso de otros. Dichos paquetes permiten modificar, analizar y visualizar las bases de datos correspondientes para lograr plasmar y representar sus componentes en el ordenador (Urdinez \& Cruz Labrín, 2020, cap. 16), valiendo, igualmente, para la creación de los mapas coropléticos ${ }^{9}$ respecto a la magnitud de las transferencias a analizar. Asimismo, tanto por ser una región funcionalmente agrícola y socialmente pobre, como también por deseo personal, se delimitan espacialmente los datos a la región de O’Higgins. Asimismo, el análisis se realizará a nivel comunal y por estrato de productor.

\footnotetext{
${ }^{8}$ Puede corresponder a obras civiles (embalses de menor tamaño, bocatomas, piscinas de acumulación de agua, entre otras) de tecnificación (riego intrapredial, riego extrapredial, entre otras) o drenaje (alcantarillado, subdrenaje superficial, subdrenaje subterráneo, entre otras) (CNR, s. f.).

${ }^{9}$ Como indica la Escuela Técnica Superior de Ingenieros en Topografía, Geodesia y Cartografía de la Universidad Politécnica de Madrid, los mapas coropléticos son "una forma de cartografiado cuantitativo utilizada para la representación de fenómenos discretos asociados a unidades de enumeración (provincias, países) a las que se aplican símbolos superficiales de acuerdo con su valor", a lo que agrega que "normalmente se realizan para obtener una idea de la distribución general de la variable cartografiada, y su función más importante suele ser la de comparar unos mapas con otros" (UPM, s. f.).
} 


\section{Distribución del presupuesto de la ley de riego}

La presentación y visualización de los resultados será a través de tablas e ilustraciones junto con una posterior descripción. A modo previo, la visualización de la distribución territorial del presupuesto agrícola da cuenta de una asignación desigual a nivel comunal, tanto en el número de proyectos presentados y en la cantidad de beneficiarios finales de los proyectos como en el monto de las transferencias respectivas. En este sentido, se postula la formación de clústeres informales (Porter, 1990, citado en Capó-Vicedo et al., 2007, pp. 4-5) en torno a las asignaciones presupuestarias de la ley de riego, representando una faceta más de las intervenciones estatales asociadas al mismo fenómeno en la década de los 90. Se evidencia la distribución heterogénea del presupuesto, patrón que se expande entre los productores de la región analizada y abre el debate respecto al rol del Ministerio de Agricultura (MINAGRI) en la construcción de un territorio más equitativo, el cual considere el desarrollo agrícola como un complemento al desarrollo de los territorios rurales y no una mera ventaja comparativa destinada a su explotación sectorial.

Para tener una visión más general, se analiza, en primera instancia, la distribución del presupuesto de la ley de riego entre las regiones del país. La tabla $1^{10}$ resume dichos montos entre los años 1990 al 2019, encontrándose expresados en UF y pesos. A su vez, se encuentran en orden decreciente respecto a la magnitud de la transferencia.

Tabla 2 Total de transferencias asociadas a la ley $\mathrm{N}^{\circ}$ 18.450. Regiones de Chile, 1990-2019

\begin{tabular}{lcc}
\hline \multicolumn{1}{c}{ Región } & Monto de las transferencias (UF) & Monto de las transferencias (\$) \\
\hline Maule & $10.520 .305,5$ & 306.540 .661 .950 \\
Coquimbo & $6.859 .581,1$ & 199.874 .474 .675 \\
O’Higgins & $4.660 .530,1$ & 135.798 .526 .345 \\
Valparaíso & $2.762 .866,2$ & 80.504 .394 .170 \\
Biobío & $2.631 .864,9$ & 76.687 .279 .456 \\
Araucanía & $2.237 .789,6$ & 65.204 .713 .948 \\
Nuble & $2.181 .941,9$ & 63.577 .423 .374 \\
Atacama & $1.641 .412,3$ & 47.827 .471 .889 \\
Metropolitana & $1.455 .651,1$ & 42.414 .762 .335 \\
Arica y Parinacota & $1.124 .371,7$ & 32.761 .941 .720 \\
Los Lagos & $701.016,1$ & 20.426 .205 .956 \\
Los Ríos & $685.660,4$ & 19.978 .773 .609 \\
Antofagasta & $360.736,0$ & 10.511 .127 .025 \\
Aysén & $230.642,1$ & 6.720 .450 .384 \\
Tarapacá & $190.832,6$ & 5.560 .479 .133 \\
Magallanes & $117.490,7$ & 3.423 .444 .891 \\
\hline Total & $\mathbf{3 8 . 3 6 2 . 6 9 2 , 4}$ & $\mathbf{1 . 1 1 7 . 8 1 2 . 1 3 0 . 8 6 0}$
\end{tabular}

\footnotetext{
${ }^{10}$ Todas las tablas e ilustraciones presentadas contienen mínimas alteraciones debido a que se encuentran aproximadas, por un lado, y a que se han creado distintas regiones durante el período analizado, como el caso de las regiones de Arica y Parinacota y Los Ríos en el año 2007 o el caso de la región del Ñuble en el año 2018, por otro. Sin embargo, estas últimas no inciden en el artículo ni en sus resultados.
} 
De la anterior tabla se puede apreciar que los productores de la región a estudiar recibieron un total de 135.000 millones de pesos por concepto de la ley de riego, a la vez que los criterios en la distribución de la ley pueden ser fácilmente identificables. Tanto el criterio de ventaja comparativa y de pobreza resultan ser determinantes a la hora de distribuir el presupuesto de la ley de riego. No obstante, no pueden perderse de vista las variables que determinan que un proyecto obtenga, en definitiva, el subsidio. Dichas variables no pasan por los criterios anteriores, sino que se relacionan con el costo total de ejecución del proyecto, con la superficie de nuevo riego que incorpora el proyecto -o su equivalente cuando se trate de un proyecto que mejora la seguridad de riego, como las obras civiles- y el aporte del beneficiario para su construcción (CNR, s. f.).

Por su parte, la tabla 2 muestra las transferencias asociadas a la ley de riego distinguiendo las comunas de la región de O’Higgins entre los años 1990 al 2019. El monto de las transferencias se expresa tanto en UF como en pesos chilenos, encontrándose ordenados de forma decreciente. Asimismo, se vislumbra la cantidad total de productores de una comuna que resultaron beneficiados en el rango de tiempo estudiado, lo que indica que un productor pudo resultar beneficiado múltiples veces. Por último, se expresa el monto de transferencias promedio o per cápita percibido por los productores de una comuna en pesos chilenos.

Tabla 3 Total de transferencias asociadas a la ley N 18.450. Comunas de la región de O'Higgins, 1990-2019

\begin{tabular}{lcccc}
\hline \multicolumn{1}{c}{ Comuna } & $\begin{array}{c}\text { Monto total de las } \\
\text { transferencias (UF) }\end{array}$ & $\begin{array}{c}\text { Monto total de las } \\
\text { transferencias (\$) }\end{array}$ & $\begin{array}{c}\text { Cantidad de } \\
\text { beneficiarios }\end{array}$ & $\begin{array}{c}\text { Monto promedio de } \\
\text { 1as }\end{array}$ transferencias ${ }^{\mathbf{(}} \mathbf{\text { \$) }}$ \\
\hline Chimbarongo & $545.979,17$ & 15.908 .741 .055 & 5.410 & 2.940 .617 \\
Pichidegua & $369.754,70$ & 10.773 .912 .449 & 9.637 & 1.117 .973 \\
San Vicente & $365.377,59$ & 10.646 .372 .217 & 11.399 & 933.974 \\
Santa Cruz & $290.768,90$ & 8.472 .424 .208 & 5.587 & 1.516 .453 \\
San Fernando & $270.348,66$ & 7.877 .419 .255 & 3.486 & 2.259 .730 \\
Rengo & $242.870,66$ & 7.076 .765 .291 & 1.879 & 3.766 .240 \\
Las Cabras & $231.383,57$ & 6.742 .054 .463 & 4.441 & 1.518 .138 \\
Nancagua & $212.831,61$ & 6.201 .487 .452 & 1.560 & 3.975 .312 \\
Peralillo & $208.464,99$ & 6.074 .252 .879 & 829 & 7.327 .204 \\
Lolol & $182.837,20$ & 5.327 .510 .334 & 188 & 28.337 .820 \\
Palmilla & $165.496,74$ & 4.822 .244 .010 & 1.508 & 3.197 .774 \\
Chépica & $149.161,66$ & 4.346 .272 .449 & 1.935 & 2.246 .135 \\
Machalí & $137.587,82$ & 4.009 .033 .899 & 8.912 & 449.846 \\
Marchigüe & $136.971,30$ & 3.991 .069 .739 & 169 & 23.615 .797 \\
Doñihue & $126.908,67$ & 3.697 .864 .826 & 2.586 & 1.429 .955 \\
Requínoa & $109.104,18$ & 3.179 .077 .597 & 2.399 & 1.325 .167 \\
Mostazal & $107.347,02$ & 3.127 .877 .469 & 1.630 & 1.918 .943 \\
Malloa & $95.503,73$ & 2.782 .787 .685 & 1.128 & 2.467 .010 \\
Placilla & $90.963,72$ & 2.650 .500 .873 & 672 & 3.944 .197 \\
La Estrella & $78.503,63$ & 2.287 .438 .771 & 40 & 57.185 .969 \\
Codegua & $77.760,20$ & 2.265 .776 .708 & 390 & 5.809 .683 \\
Peumo & $74.943,74$ & 2.183 .710 .696 & 1.583 & 1.379 .476 \\
Coltauco & $72.401,91$ & 2.109 .646 .854 & 2.068 & 1.020 .138 \\
Rancagua & $67.533,64$ & 1.967 .795 .202 & 1.114 & 1.766 .423
\end{tabular}




\begin{tabular}{lcccc} 
Pumanque & $63.435,38$ & 1.848 .380 .102 & 45 & 41.075 .113 \\
Graneros & $54.888,23$ & 1.599 .333 .246 & 387 & 4.132 .644 \\
Quinta de & $46.349,17$ & 1.350 .522 .115 & 556 & 2.428 .996 \\
Tilcoco & $31.253,48$ & 910.663 .900 & 327 & 2.784 .904 \\
Olivar & $27.653,68$ & 805.772 .928 & 17 & 47.398 .407 \\
Litueche & $14.149,65$ & 412.292 .502 & 201 & 2.051 .206 \\
Coinco & $6.640,47$ & 193.490 .015 & 184 & 1.051 .576 \\
Navidad & $3.519,33$ & 102.546 .238 & 5 & 20.509 .247 \\
Paredones & $1.835,71$ & 53.488 .918 & 1 & 53.488 .918 \\
Pichilemu & $\mathbf{4 . 6 6 0 . 5 3 0 , 1 1}$ & $\mathbf{1 3 5 . 7 9 8 . 5 2 6 . 3 4 5}$ & $\mathbf{7 2 . 2 7 3}$ & - \\
\hline Total & &
\end{tabular}

a Esta medición no es fidedigna por cuanto la distribución del presupuesto es asimétrica. Por ello, es de esperar una alta desviación estándar que restaría veracidad a la medición per cápita. Sin embargo, a falta de un análisis estadístico más riguroso, conviene estudiar las transferencias promedio.

La tabla anterior deja en evidencia la inequidad tanto en la distribución del presupuesto hacia los productores de las comunas de la región como en los beneficiarios finales de la ley de riego. En este sentido, la comuna de Pichilemu es aquella que percibió la menor cantidad de recursos, mientras que su productor percibió el mayor beneficio pecuniario individualmente. A su vez, la comuna de Chimbarongo recibió, en total, cerca de 300 veces más recursos monetarios que Pichilemu, pero el monto percibido individualmente es mucho menos. Ambos elementos se entrelazan en la medida que el presupuesto percibido se ve determinado por la concentración de agricultores en una comuna que presentan un proyecto asociado a dicha ley. Esta misma razón aplica al cálculo per cápita, al verse influenciado por la cantidad de beneficiarios finales. En este punto se vuelve relevante visualizar el resultado en el espacio de las transferencias asociadas a las comunas. Para ello, la ilustración 1 contiene el mapeo de la anterior tabla.

\section{Ilustración 1}

Distribución espacial de las transferencias asociadas a la ley $\mathrm{N}^{\circ} 18.450$

Comunas de la región de O’Higgins, 1990-2019

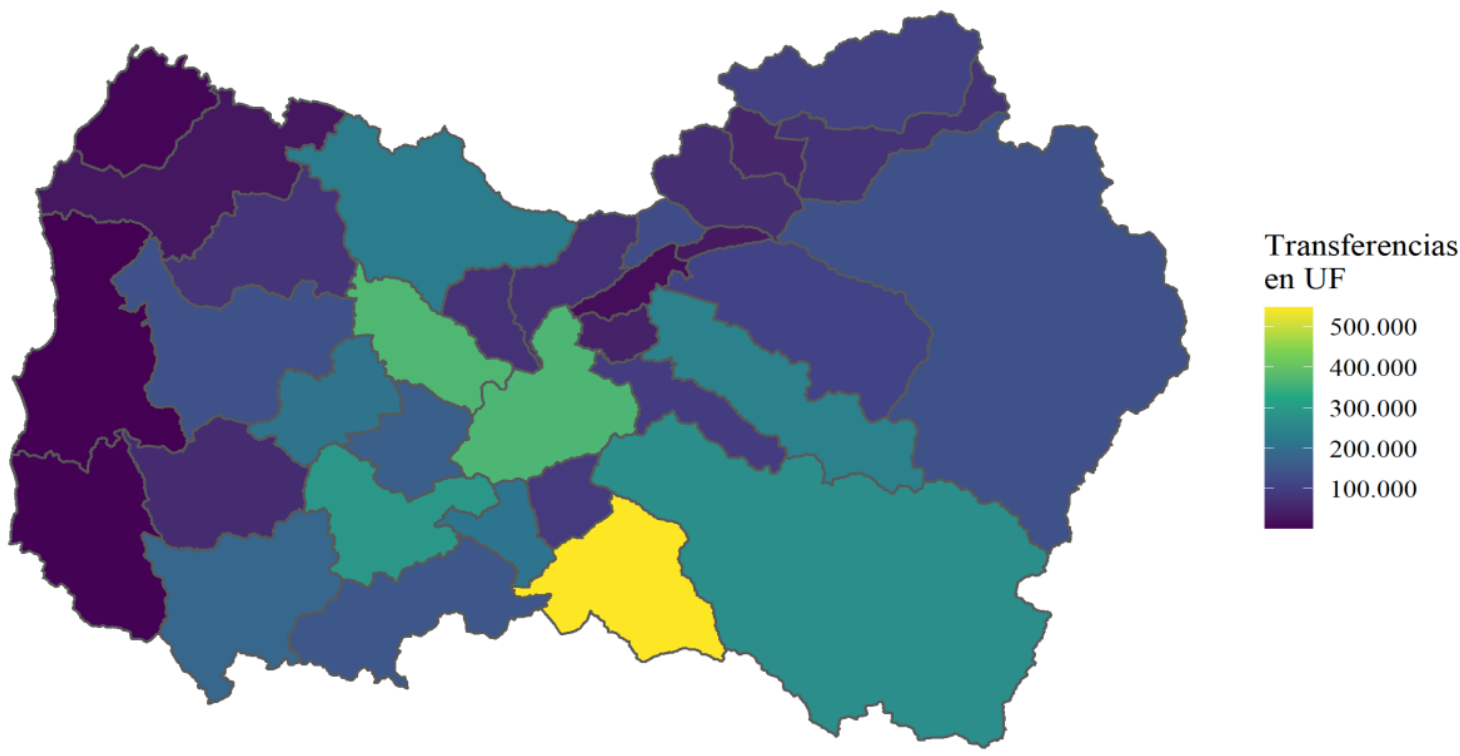


La ilustración permite evidenciar patrones geográficos en la distribución del presupuesto, ello debido a la existencia de comunas que sirven de polos de alta y baja concentración tanto de subsidios como de agricultores. Por ello, resalta la proximidad geográfica de los mismos, que es la que, en definitiva, permite afirmar la existencia de uno de los elementos de la noción de "polo", al suponer la concentración de un fenómeno en una misma locación.

En la región, el principal polo de alta concentración presupuestaria, presente en el centro del territorio, está conformado por las comunas de Chimbarongo, Pichidegua y San Vicente de Tagua Tagua. En el sentido contrario, los polos de baja asignación presupuestaria son dos. Uno de ellos, ubicado en la zona costera de la región, contiene a las comunas de Litueche, Navidad, Paredones y Pichilemu. El segundo de ellos, ubicado en la zona cordillerana del territorio, contiene a las comunas de Machalí, Codegua, Graneros, Mostazal y Requínoa, expandiéndose hacia el interior de la región.

Fuera de lo anterior, y en pos de realizar un análisis más específico en torno a los productores beneficiarios de los subsidios asociados a la ley de riego, es necesario atender al nivel de estratos de productores que percibieron la transferencia, ello a fin de reconocer patrones o conductas que incidieran en la justa y equitativa distribución del presupuesto. La tabla 4 contiene los montos totales de las transferencias asociadas a la ley de riego y los montos per cápita de las mismas transferencias según los estratos, encontrándose expresadas en UF y abarcando entre los años 1990 al 2019.

Tabla 4 Total de transferencias asociadas a estratos de la ley N 18.450. Región de O'Higgins, 19902019

\begin{tabular}{lcccc}
\hline \multicolumn{1}{c}{$\begin{array}{c}\text { Estrato de } \\
\text { productor }\end{array}$} & $\begin{array}{c}\text { Monto total de } \\
\text { transferencias } \\
(\mathbf{U F})\end{array}$ & $\begin{array}{c}\text { Monto total de } \\
\text { transferencias (\$) }\end{array}$ & $\begin{array}{c}\text { Cantidad de } \\
\text { beneficiarios }\end{array}$ & $\begin{array}{c}\text { Monto promedio de } \\
\text { transferencias (\$) }\end{array}$ \\
\hline $\begin{array}{l}\text { Organizaciones de } \\
\text { Pequeños Usuarios }\end{array}$ & $1.912 .977,1$ & 55.740 .326 .448 & 63.472 & 878.187 \\
$\begin{array}{l}\text { Empresario } \\
\text { Mediano }\end{array}$ & $1.466 .471,8$ & 42.730 .055 .891 & 1.495 & 28.581 .977 \\
Pequeño & $748.372,8$ & 21.806 .086 .938 & 958 & 22.762 .094 \\
Empresario & $210.839,5$ & 6.143 .442 .517 & 214 & 28.707 .675 \\
Gran Empresario & $159.391,3$ & 4.644 .343 .117 & 5.756 & 806.869 \\
Organizaciones de & $158.385,2$ & 4.615 .029 .123 & 322 & 14.332 .388 \\
Usuarios & $\mathbf{4 . 6 5 6 . 4 3 7 , \mathbf { 8 } ^ { \mathrm { a } }}$ & $\mathbf{1 3 5 . 6 7 9 . 2 8 4 . 0 3 4}$ & $\mathbf{7 2 . 2 1 7}$ & - \\
\hline Productor INDAP & Total & & & \\
\hline
\end{tabular}

${ }^{\text {a }}$ La diferencia en los totales respecto a la tabla 2 y 3 se debe a que la base de datos omite la información respecto a los estratos de productor de ciertos proyectos adjudicados, no pudiendo expresarse en la presente tabla. En total, representan la diferencia de $4.092 \mathrm{UF}$.

De las anteriores ilustraciones se desprende el predominio de las Organizaciones de Pequeños Usuarios del Agua respecto al monto total de transferencias percibidas, seguidas por los estratos de Empresarios Medianos y Pequeños Empresarios. Por su parte, las menores transferencias totales son percibidas por los estratos de Grandes Empresarios, las Organizaciones de Usuarios del Agua y los Productores INDAP.

$\mathrm{Si}$ bien la diferencia entre dichos grupos es bastante amplia y definida, los Empresarios Medianos y Grandes Empresarios resultan ser los más beneficiados respecto a la medición per cápita. Asimismo, resalta la notable disminución proporcional del segmento de las OPUA, ya que, al ser entes 
asociativos, los proyectos que se adjudican benefician a grandes cantidades de productores, lo que se traduce en transferencias mínimas de dinero a cada beneficiario tras el cálculo per cápita.

Frente a ello, es dable analizar la distribución territorial de dichas transferencias considerando al estrato de productores indicado. Las dos ilustraciones siguientes -4 y 5- presentan las transferencias territoriales según el tipo de productor, considerando, la primera de ellas, el total de las transferencias y, la segunda, las transferencias per cápita. Asimismo, los montos se encuentran expresados en UF, considerándose las transferencias realizadas entre los años 1990 al 2019. 
Ilustración 2

Total de transferencias asociadas a la ley $\mathrm{N}^{\circ} 18.450$ seguin estrato de productores

Comunas dec a region de ()'Higgins, 1990-20119

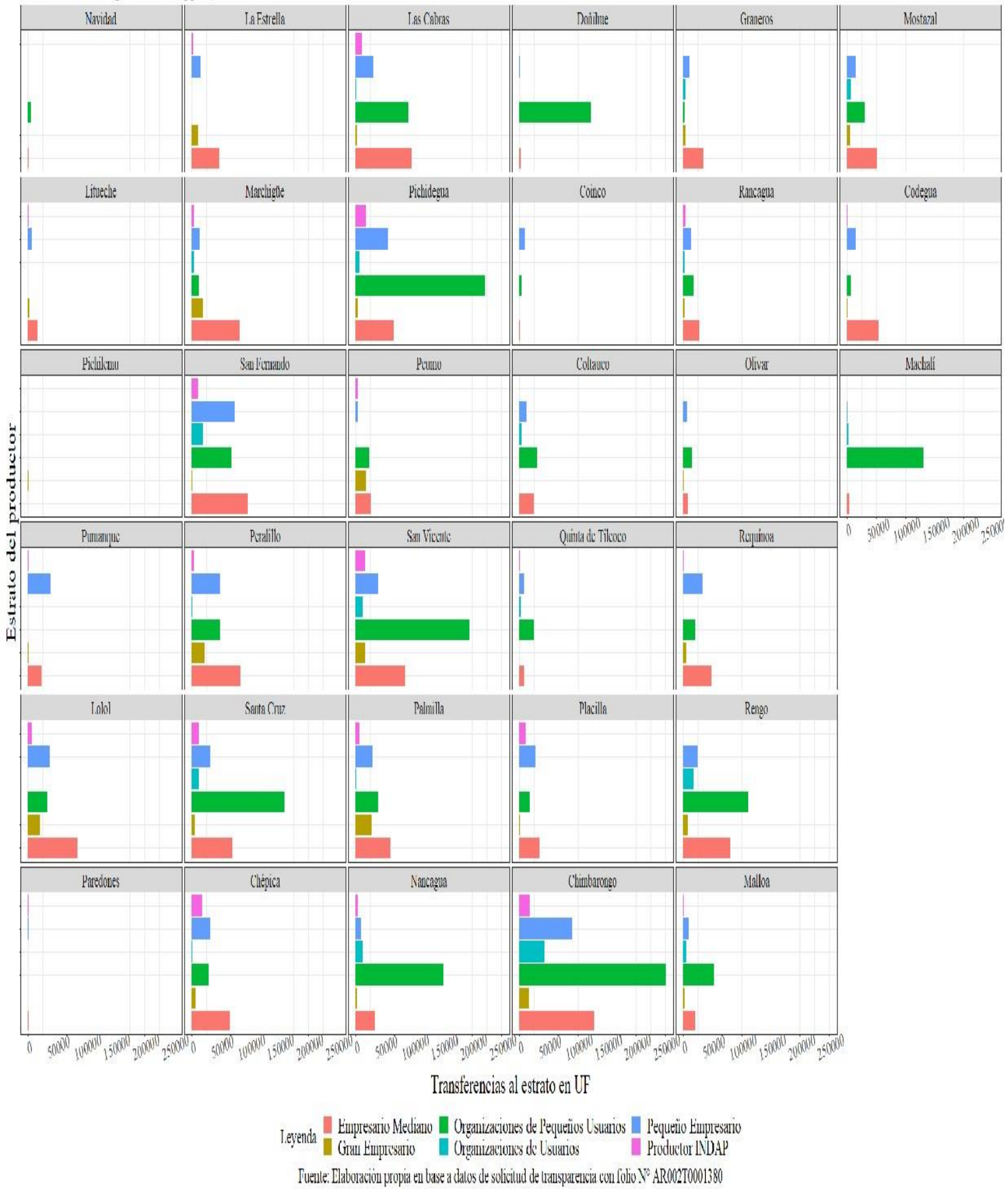


Ilusitración 3

Total de transferencias asociadas a la ley $\mathrm{N}^{0} 18.450$ segiun estrato de productores

Comunas de la region de OHigogiss, 1990-2019

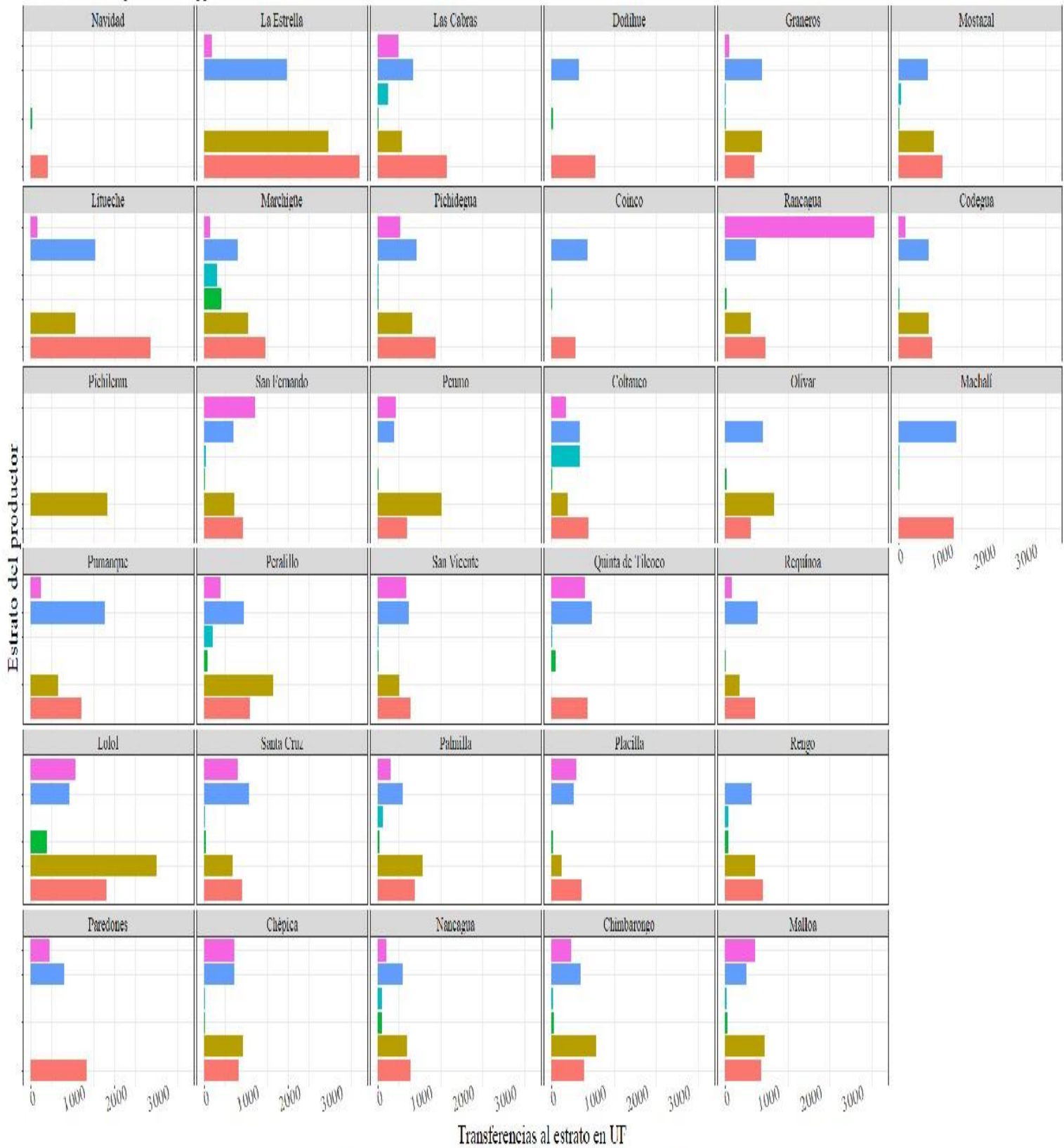

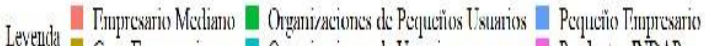

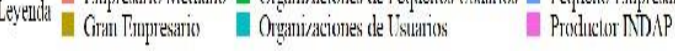

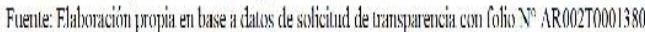


Respecto a la ilustración 2, esta zonifica las comunas a las que pertenecen los productores que recibieron financiamiento categorizando las transferencias según los estratos correspondientes. Se puede apreciar como los integrantes de las OPUA están concentrados en comunas como Chimbarongo, Doñihue, Pichidegua, Machalí, San Vicente de Tagua Tagua, Santa Cruz, Rengo y Nancagua. Por su parte, los Empresarios Medianos, si bien perciben menores transferencias en comparación a las OPUA, logran mayor constancia a lo largo del territorio, siendo especialmente relevantes en comunas como Chimbarongo, Las Cabras, Marchigüe, San Fernando, Peralillo, San Vicente de Tagua Tagua y Lolol. El tercer estrato con el mayor monto asignado corresponde a los Pequeños Empresarios, destacando en las comunas de Pichidegua, San Fernando y Chimbarongo, encontrándose ramificados a lo largo del territorio regional. Sin embargo, respecto a las transferencias per cápita, los montos cambian sustancialmente. La ilustración 3 muestra la asignación promedio transferida a cada beneficiario según el estrato del productor que presentó el proyecto. Si bien los proyectos de mayor cuantía monetaria son adjudicados por las OPUA, quienes perciben individualmente los mayores beneficios pecuniarios son los productores pertenecientes a los estratos de Pequeños, Medianos y Grandes Empresarios.

Estos resultados dan cuenta de una distribución heterogénea del presupuesto de la ley de riego, el cual se concentra en proyectos adjudicados a determinadas comunas y a determinados estratos de productores de la región de O’Higgins. Asimismo, se tiene uno de los elementos que permiten distinguir la formación de polos de concentración de las asignaciones que, en este caso, refiere a la aglomeración espacial de las transferencias a los agricultores.

\section{Discusión}

Mientras se han formado polos informales de asignaciones monetarias en las comunas de la región de O’Higgins, aquellas transferencias realizadas hacia los Medianos y Grandes Empresarios no encuentran justificación alguna, entendiéndose más como un privilegio que como una intervención estatal ante una falla de mercado (DIPRES, 2019). En este sentido, destaca la proximidad geográfica en la concentración de las asignaciones, fenómeno que amerita un análisis de autocorrelación espacial que indague en sus causas a fin de avanzar en la comprensión cabal del mismo (Bivand, Pebesma y Gómez-Rubio, 2013, citados en Urdinez \& Cruz Labrín, 2020, cap. 16).

Llegado a esta instancia, es necesario aclarar dos puntos. Primero, este patrón distributivo del presupuesto puede repetirse en multitud de otras políticas. Dicho conocimiento no entregaría más información por sí mismo, mucho menos indicaría que el gasto representase mayor eficacia, eficiencia ni efectividad respecto a los objetivos de la misma o de la problemática a tratar, como tampoco indicaría una mejora en la calidad de vida de los beneficiarios o la generación de valor público. Lo que si es factible de afirmar es la importancia que las instituciones le otorgan al territorio mediante la distribución presupuestaria, y ello no sólo debiera suponerse respecto a la magnitud de las transferencias, sino respecto a los mecanismos que existen para salvaguardar la integridad de aquellos territorios rezagados al momento de dicha distribución.

Lo anterior conduce al segundo punto, y refiere al rol que tiene el Estado en la construcción y moldeamiento tanto del territorio como de la estructura socioeconómica rural, en este caso. Los objetivos de las políticas agrícolas impulsadas por el MINAGRI han referido, en esencia, al aumento de la productividad y competitividad de los predios agrícolas, a la preservación de comunidades rurales y a la protección del medioambiente (ODEPA, 2019). Sin embargo, como bien acredita la evaluación de la DIPRES sobre la ley $\mathrm{N}^{\circ}$ 18.450, dichas normativas, si bien se encuentran orientadas al aumento de la productividad y competencia de los agricultores, no consideran mecanismos que permitan asegurar la preservación de las comunidades o la protección del medioambiente.

En este sentido, destaca la promulgación y publicación en el año 2020 del Decreto $\mathrm{N}^{\circ}$ 19, que aprueba la Política Nacional de Desarrollo Rural, la cual es percibida como una ventana de oportunidad para 
esclarecer los cuestionamientos e inquietudes respecto a este punto. En la medida que dicha normativa articule los polos informales mencionados, permitirá avanzar tanto en la clusterización de los productores menores como también en la implementación del paradigma de nueva ruralidad en nuestro país. Ante ello, el sector agrícola contará con bases más sólidas que le permitirán progresar desde una estrategia productivista a una modernizadora, la cual no privilegie netamente la agricultura de exportación, sino una en la cual las redes formadas entre los distintos sectores rurales y agrícolas interactúen entre sí, resultando en elementos capaces de reorientar el perfil del modelo de desarrollo, a fin de integrar y coordinar las acciones entre las políticas y sus actores en sintonía con las necesidades locales del territorio ${ }^{11}$.

A pesar de la existencia de acciones que pudiesen avanzar en la conjunción de actores y políticas de la agricultura, y a sabiendas del estado actual del rubro, se postula que el presupuesto de la ley $\mathrm{N}^{\circ} 18.450$ se encuentra bloqueado -locked-in-, concentrado en unos pocos productores de unas pocas comunas, lo que dificulta la llegada al territorio de la institucionalidad agrícola en desmedro de sus objetivos primordiales.

Fuera de lo anterior, esta discusión sobre la pertinencia de progresar desde polos a clústeres en la agroindustria no cuenta con datos que abalen su factibilidad. La aplicación de la teoría a las prácticas agrícolas requeriría de un estudio acabado que promueva dicha estrategia. Sin embargo, el clúster sería una base óptima -no la única- para solventar algunas problemáticas claves del sector. A saber, una forma en la que la diversificación productiva podría verse potenciada refiere a la articulación de empresas de generación de conocimiento y a agricultores con el fin de innovar tanto en los procesos productivos como en los productos finales; mitigar la disminución en el tiempo de la pequeña agricultura y la agricultura familiar -o preservar las comunidades- puede pasar por la creación de redes que vayan más allá de lo productivo, otorgando un sistema de seguridad social que tenga como uno de sus ejes centrales asegurar la competitividad en el mercado y, desde ahí, velar por el bienestar integral de los mismos; proteger el medioambiente en el que se desenvuelve el rubro puede encontrar una arista en la plausible descongestión que tendrían los sistemas hídricos y terrestres tras progresas desde una estrategia intensificadora a una de modernización e innovación en los procesos productivos; entre otras. En definitiva, formalizar los polos detectados desde la institucionalidad agraria y permitir que los agricultores formen redes entre ellos y con empresas de generación de conocimiento refiere a una cuestión más allá de la mera ley de riego, implica un cambio de paradigma en la manera que se desempeña el rubro en nuestro país, escapando del objetivo de este artículo.

\section{Conclusiones}

La concentración de las asignaciones en determinados territorios y productores se relaciona con que los arreglos institucionales, al menos de la ley $\mathrm{N}^{\circ} 18.450$, no orientan el presupuesto según criterios de equidad social o según el nivel de rezago de determinados territorios. Mucho menos procura coordinar las acciones ministeriales en pos de solventar el fenómeno subyacente a la realidad rural de nuestro país -esto es, la pobreza- sino que su diseño conduce a la mencionada concentración. Algunas posibles causas pueden referir a la concentración de productores agrícolas en una determinada locación, al desconocimiento de los productores sobre los concursos públicos disponibles, a la no utilización de la tierra o su explotación en otro rubro, a la matriz productiva comunal o zonal, e incluso a las bondades geográficas, climatológicas o hidrológicas de la zona, entre otras. Sumado, en el caso del polo de asignación cercano a Chimbarongo, la expansión de los productores -y la postulación de sus proyectos- puede estar ligada tanto al embalse Convento Viejo, en la misma comuna, como al auge vitivinícola en la provincia de Colchagua, lo que puede explicar tanto el mayor gasto en el eje Chimbarongo-Santa Cruz como los altos grados de asociatividad y vínculos.

Respecto a la teoría planteada, se ha vislumbrado someramente lo que implicaría la aplicación de la teoría de polos y clústeres sobre la agricultura nacional, concluyéndose que, si bien existen indicios de la

11 Para más información respecto a esta perspectiva, se recomienda revisar el paradigma de "Nueva Ruralidad" que, si bien le resta centralidad a la agricultura en el mundo rural, moderniza y repiensa sus elementos claves. 
formación de polos en la región estudiada, avanzar en la clusterización para alcanzar los beneficios de innovación y articulación entre los productores agrícolas implicaría, por un lado, contar con un estudio que avalase la factibilidad de tal acción y, por otro, reformar estructuralmente tanto los arreglos institucionales asociados como la estrategia actual impulsada por los gobiernos del país.

Respecto a los resultados obtenidos, se concluye que existen argumentos para sostener que la distribución territorial del presupuesto de la ley $\mathrm{N}^{\circ} 18.450$ es heterogénea, ya sea a nivel comunal o según los estratos de productores establecidos. Tanto los patrones de asignación que forman los polos de alta y baja asignación monetaria como las transferencias hacia los Medianos y Grandes Empresarios dan cuenta que el actuar de las instituciones tendió a concentrar el presupuesto agrícola en el territorio y en el patrimonio de los productores. Sin embargo, ello no permite comprender en demasía lo que realmente implica una distribución heterogénea del presupuesto, ya que no se consideraron elementos como el impacto en la productividad del rubro, en la calidad de vida de las personas o en el medioambiente en sí, lo que pudiese justificar o agravar los resultados presentados.

\section{Bibliografía}

Anríquez, G., Foster, W., Ortega, J., Falconi, C., \& De Salvo, C. P. (2016). Public Expenditures and the Performance of Latin American and Caribbean Agriculture. Inter-American Development Bank. https://doi.org/10.18235/0000510

Banco Mundial. (2005). Beyond the City: The Rural Contribution to Development. Banco Mundial. https://openknowledge.worldbank.org/bitstream/handle/10986/7328/32333.pdf?sequence=1\&isAll owed $=\mathrm{y}$

BCN. (2018). Principales contenidos y alcances de la Ley $\mathrm{N}^{\circ} 18.450$ (Ley de Riego). https://www.camara.cl/verDoc.aspx?prmTIPO=DOCUMENTOCOMUNICACIONCUENTA\&pr $\underline{\mathrm{mID}}=75068$

Boudeville, J. R. (1967). Problems of Regional Economic Planning. The Economic Journal, 77(307), 629-630. https://doi.org/10.2307/2229009

Capó-Vicedo, J., Expósito-Langa, M., \& Tomás-Miquel, J. V. (2007). Creación de Redes Interorganizativas en un Cluster Territorial. Información tecnologica, 18(5). https://doi.org/10.4067/S0718$\underline{07642007000500002}$

CEPAL. (2010). Evolución y distribución del ingreso agrícola en América Latina: Evidencia a partir de cuentas nacionales y encuestas de hogares. CEPAL. https://www.cepal.org/es/publicaciones/3794evolucion-distribucion-ingreso-agricola-america-latina-evidencia-partir-cuentas

Chapman, K. (2005). From «growth centre» to «clusten»: Restructuring, regional development, and the Teesside chemical industry. Environment and Planning A, 37(4), 597-615. https://doi.org/10.1068/a36270

CNR. (s. f.). Preguntas frecuentes. Recuperado 4 de enero de 2021, de https://www.cnr.gob.cl/atencionciudadana/preguntas-frecuentes/

Coraggio, J. L. (1972). Hacia una revisión de la teoría de los polos de desarrollo. Revista EURE - Revista de Estudios Urbano Regionales, 2(4), Article 4. https://doi.org/10.7764/820

DIPRES. (2019). Informe Final de Evaluación de Programas Gubernamentales (EPG) Programas Obras de Riego Menores y Medianas ley 18.450 y Fomento al Riego. http://www.dipres.gob.cl/597/articles189313 informe final.pdf

Feser, E. J. (1998). Old and New Theories of Industry Clusters. En Clusters and Regional Specialisation: On Geography, Technology, and Networks (pp. 18-40).

https://www.researchgate.net/publication/273754939 Clusters and Regional Specialisation On Geograph y Technology and Networks 
Figueroa-Sterquel, R., Chia, E., \& Huerta, P. (2016). Estudio del Clúster como un Instrumento de Gobernanza Territorial en Chile: El Caso de la Palta en la Región de Valparaíso. Revista geográfica de Valparaíso, 53, 121-141.

https://www.google.com/url?sa=t\&rct=j\&q=\&esrc=s\&source=web\&cd=\&ved=2ahUKEwjVisqXs 7tAhW LLLkGHfLzC7kQFjAMegQIExAC\&url=http $\% 3 \mathrm{~A} \% 2 \mathrm{~F} \% 2 \mathrm{Fwww}$.revistageografica.cl $\% 2$ Findex.php $\% 2 \mathrm{Frevg}$ eo $\% 2$ Farticle $\% 2$ Fdownload $\% 2 F 8 \% 2 F 8 \& u s g=A O v V a w 17 \mathrm{Ky} 1 \mathrm{FZkMBeXXBAkTtxCX}$ -

Hirschman, A. O., \& Sirkin, G. (1958). Investment Criteria and Capital Intensity Once Again. The Quarterly Journal of Economics, 72(3), 469-471. https://doi.org/10.2307/1882237

Lasuen, J. R. (1969). On Growth Poles. Urban Studies, 6(2), 137-161.

https://doi.org/10.1080/00420986920080231

Martin, R., \& Sunley, P. (2003). Deconstructing clusters: Chaotic concept or policy panacea? Journal of Economic Geography, 3(1), 5-35. https://doi.org/10.1093/jeg/3.1.5

Myrdal, G. (1957). Economic Theory and Underdeveloped Regions.

http://duncankennedy.net/documents/Is-Pal/SecondSyllabus/G. \%20Myrdal, \%20Economic $\% 20$ Theory $\% 20$ and $\% 20$ Underdeveloped $\% 20$ Regions.pdf

ODEPA. (2019). Nivel y composición del apoyo del Estado a la agricultura en Chile durante el periodo 19902017. https://www.odepa.gob.cl/wp-content/uploads/2019/05/EstudiosEstrategico2-1.pdf

Perroux, F. (1950). Economic Space: Theory and Applications1. The Quarterly Journal of Economics, 64(1), 89104. https://doi.org/10.2307/1881960

Porter, M. (1990). The Competitive Advantage of Nations. Harvard Business Review, 68(2), 73-93. https://hbr.org/1990/03/the-competitive-advantage-of-nations

Porter, M. (1995). The Competitive Advantage of the Inner-City. Harvard Business Review, 73(3), 55-71. https://hbr.org/1995/05/the-competitive-advantage-of-the-inner-city

Porter, M. (1996). Competitive advantage, agglomeration economies, and regional policy. International Regional Science Review, 19(1-2), 85-90. https://doi.org/10.1177/016001769601900208

Porter, M. (1998). Clusters and the New Economics of Competition. Harvard Business Review, 77-90. http://marasbiber.com/wp-content/uploads/2018/05/Michael-E.-Porter-Cluster-Reading.pdf

Porter, M. (2000). Location, competition, and economic development: Local clusters in a global economy. Economic Development Quarterly, 14(1), 15-34. https://doi.org/10.1177/089124240001400105

Smékalová, L., Hrabinová, Š., \& Habuda, M. (2014). Spatial distribution of competitiveness support in the Slovakia in relation to growth poles and small and medium enterprises. https://www.semanticscholar.org/paper/Spatial-distribution-of-competitiveness-support-inSmékalová-Hrabinová/b756aedd2627a275b0faf89f1d2861ae9b115e34

UPM. (s. f.). Cartografía Cuantitativa: Coropletas. Recuperado 4 de enero de 2021, de http://pdi.topografia.upm.es/mab/tematica/htmls/coropletas.html\#resumen

Urdinez, F., \& Cruz Labrín, A. (2020). AnalizaR Datos Políticos. https://arcruz0.github.io/libroadp/ 